\title{
LAS COMPETENCIAS CIENTÍFICAS Y LA MULTICULTURALIDAD EN LA COLOMBIA DEL SIGLO XXI
}

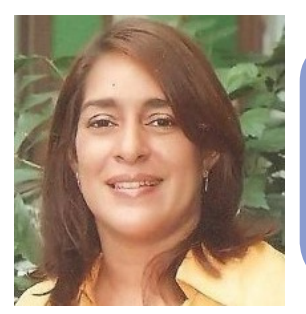

Milfred Coronado ${ }^{1}$

Doctoranda en Ciencias de la Educación de la Universidad Metropolitana de Educación, Ciencia y Tecnología de Panamá (UMECIT) mcoronadoborja@hotmail.com

\section{RESUMEN}

El propósito de este documento es reflexionar sobre la temática alusiva a las competencias científicas y la multiculturalidad, en la Colombia del siglo XXI. A través de una revisión teórico conceptual y un acercamiento espacio-temporal se logra hacer un análisis del desarrollo de las competencias científicas en escenarios multiculturales en el país del café, del carbón y la esmeralda, en particular en la Región Caribe colombiana. El escrito propone el desarrollo de un currículo que observe la localidad pero que no se aleje de lo regional y nacional, como también que sea flexible, holístico, incluyente, entre otros. A través de él se generará experiencias significativas donde el estudiante inmerso en aulas multiculturales desarrolle también competencias científicas respetando los presaberes de estos y las investigaciones que realizan sobre su comunidad y contexto. Se hace alusión al pensamiento y a los conocimientos profesionales de los docentes porque son ellos en definitiva quienes orientan el proceso educativo y de la intencionalidad de ellos dependen el avance de los discentes en temas tan actuales como las competencias científicas y la multiculturalidad. Al final del documento se hacen algunas sugerencias dirigidas principalmente a la cualificación del maestro sobre la temática del artículo y la pertinencia de innovar en la simbiosis entre cultura y ciencia.

Palabras clave: Competencias científicas, multiculturalidad, enseñanza de las ciencias naturales, praxis profesional del docente, currículo

\footnotetext{
1 Licenciada en Ciencias de la Educación. Esp. Biología y Química, Especialista en la Enseñanza de las Ciencias Naturales, de la Universidad del Atlántico. Magíster en Educación, con énfasis en Cognición de la Universidad del Norte. Doctoranda en Ciencias de la Educación, de la Universidad Metropolitana de Educación, Ciencia y Tecnología de Panamá (UMECIT). mcoronadoborja@hotmail.com.
} 


\title{
SCIENTIFIC COMPETENCES AND MULTICULTURALITY IN COLOMBIA OF THE 21ST CENTURY
}

\begin{abstract}
The purpose of this document is to reflect on the subject allusive to scientific competences and multiculturalism, in the Colombia of the XXI century.Through a conceptual theoretical review and a spatial-temporal approach, an analysis of the development of scientific competences in multicultural settings in the country of coffee, coal and emerald is achieved, particularly in the Colombian Caribbean Region. The paper proposes the development of a curriculum that observes the locality but does not stray from the regional and national, as well as that is flexible, holistic, inclusive, among others. Through it, significant experiences will be generated where the student immersed in multicultural classrooms also develop scientific competencies respecting the presabers of these and the research they carry out on their community and context. Reference is made to the professional thinking and knowledge of teachers because it is they who ultimately guide the educational process and the intentionality of them depend on the progress of learners in topics as current as scientific competences and the multiculturalism. At the end of the document some suggestions are made mainly aimed at the qualification of the teacher on the topic of the article and the relevance of innovating in the symbiosis between culture and science.
\end{abstract}

Key words: Scientific competences, multiculturalism, teaching of natural sciences, professional praxis of the teacher, curriculum

\section{INTRODUCCIÓN}

"Desarrollar competencias científicas, implica observar: conocimiento, habilidades y valores, es decir, Saber, Hacer y Ser." Quintanilla.

En un mundo tan convulsionado por fenómenos como la globalización, la internacionalización de los procesos sociales y recientemente pandemias como el covid-19, asoma con mucha relevancia un sector que es fundamental para lograr conglomerados humanos capaces de producir los cambios y las adaptaciones requeridas antes las situaciones mencionadas: la educación.

La educación como elemento básico de cambio en una sociedad, no es ajena a ciertos factores culturales y es direccionada en la mayoría de los países para empoderar tres (3) dimensiones específicas como menciona Quintanilla “el saber, hacer y ser”. 
El actual documento que invita al lector adentrarse a una propuesta de complementariedad entre las competencias científicas y la multiculturalidad en la educación colombiana, surge como respuesta la problemática de formar niños, niñas y jóvenes con características particulares consecuencia de una civilización impactada por las tics, las economías interdependientes, la sociedad del conocimiento y la interiorización de subculturas o culturas foráneas en una mayoritaria que en momentos desea sobresalir y "apagar" o inhibir aquellas consideradas externas a sus intereses o necesidades.

Colombia como país pluriétnico y multicultural no se aparta de lo referenciado hasta aquí asume políticas y una legislación hacia el desarrollo de competencias y de la aceptación de la multiculturalidad; referentes que se quedan en papel, la mayoría de los casos y distan de la realidad durante la praxis educativa.

A través de estas líneas se propone lograr una reflexión teórico-conceptual sobre las competencias científicas y la multiculturalidad en la enseñanza de las ciencias naturales a la luz de una práctica docente, la cual es fundamental y determinante en el proceso educativo. No se desea dogmatizar sobre los temas propuestos por el contrario se busca generar un proceso de enriquecimiento cognitivo-colectivo donde cada lector opine sobre el documento y asuma posiciones dirigidas a clarificar sobre los tópicos de interés de este artículo en un contexto espacial como el colombiano.

\section{DESARROLLO}

Los conceptos de competencias y multiculturalidad se observan en el devenir histórico de la humanidad; en momentos no coincidentes, pero han evolucionado a través de los siglos. Con respecto al primer referente, este aludió en un inicio a la oralidad de los pueblos ancestrales como los griegos y los atenienses, sin embargo, a través del tiempo, el concepto de competencia va incursionando paulatinamente en diversos ámbitos como: la psicología (siglo XVII); en la filosofía y la sociología en el siglo XX, para luego irrumpir a nivel laboral al considerarse como un "conjunto de conocimientos y capacidades que permiten el desarrollo de la actividad profesional". (Murgas, 2015). En el campo de la educación existen muchos autores que han dejado sus aportes por escrito para ser objeto de consulta y/o revisión; actualmente en el mundo las competencias son prioritarias en el proceso educativo.

Siguiendo este orden de ideas, con relación al segundo referente conceptual, el autor (Fagade, 2015) indica que su génesis se remonta a la antigüedad griega y romana, cuando "se congregaban los mejores deportistas en los juegos olímpicos de Atenas, generándose intercambios de culturas, 
costumbres e ideas entre otros aspectos".

A partir de los años sesenta del anterior siglo se difundió el constructo de multiculturalidad, siendo Canadá uno de los primeros países de América del Norte que lo referencia ( (Bernabé, 2012) y el primero en adoptarlo como política nacional. Europa por su parte utiliza el termino en mención para referirse a los inmigrantes que integran su estado nacional. En América Latina y el Caribe, la expresión multicultural es aplicado a los pueblos de minorías étnicas (indígenas, afrodescendientes, LGBTI y ROM) respetando la formulación política de cada país. Colombia se puede considerar pionera en Suramérica al consolidarse en la nueva Constitución Política de 1991, el reconocimiento por la diversidad étnica y cultural nacional; definiéndose como un país incluyente y respetuoso de la multiculturalidad. En respuesta a esta carta magna surge la Ley General de Educación en 1994, la cual direcciona también hacia una educación multicultural entendida esta como una forma de afianzar un tipo de enseñanza-aprendizaje con unas características hacia una convivencia ciudadana sana, tolerante y equitativa.

Atendiendo a lo antes planteado surge la hipótesis que será fundamento para desarrollar un tipo de pensamiento alrededor de un proceso discursivo donde se invita a pensar y repensar sobre la misma: los espacios multiculturales son génesis o el "caldo" para afianzar o generar procesos de avances en lo que respecta a las competencias científicas.

Se desea comenzar la reflexión citando la película "Detrás de la Pizarra" (Bleckner, 2011), tomada de una historia real ocurrida en Salt Lake City, donde una docente toma una escuela improvisada en un refugio para personas sin techo, y logra mediante un acertado proceso de enseñanza desarrollar competencias científicas, artísticas, entre otras en personas de diferentes nacionalidades, estratos socioeconómicos y culturas, de tal forma que se genera el efecto Pigmalión y se superan como personas y educandos.

A lo antes planteado subyace entonces la idea de lo que es realmente relevante para poder lograr cambios significativos en los discentes, aunque se encuentren inmersos en un mismo espacioaula donde las características son la multiculturalidad y la diferencia socioeconómica.

Existen autores quienes plantean que las competencias científicas se desarrollan en la interrelación de los contextos disciplinar, multicultural y de la vida cotidiana. Estos autores son relevantes para este artículo en la medida que fundamentan la relación entre desarrollo de competencias científica o el hacer ciencia con los procesos de multiculturalidad, objetivo de estas líneas. (Arteta, J., Chona, G., Fonseca, C.,Martínez, S., Ibañez, S, 2002) 
En este orden de ideas, se comparte el concepto de los autores anteriores pero se aúna la premisa de que si se desea desarrollar competencias científicas en contextos multiculturales, los educadores están llamados a fortalecer sus competencias docentes a través de la cualificación continua y constante para que de esta forma puedan coadyuvar a la formación en la multiculturalidad, acerca de esto (Maldonado, 2016) expresa unos principios que aplican para el desarrollo de las competencias de los maestros en contextos latinoamericanos:

- La articulación de conocimientos entorno a los conocimientos locales y regionales.

- La investigación como eje pedagógico.

- La filosofía comunal como horizonte.

- La participación comunitaria en el proceso de aprendizaje

- El uso extensivo de la lengua originaria.

- Un currículo adecuado a la realidad en que se trabaja.

- La colaboración de maestros que, más que enseñar, ayudan a aprender

Los anteriores elementos establecidos por Maldonado indican al docente caminos para lograr desarrollar las competencias científicas de los discentes en espacios multiculturales los cuales son fundamentales en la praxis profesional del educador y por lo tanto prioritario en el establecimiento de un proceso didáctico en la enseñanza de las ciencias.

Siguiendo el hilo conductor de estas líneas, la premisa de educar a partir de los preconceptos de los alumnos, de la realidad que viven los mismos y las investigaciones que realizan los educandos en sus contextos; fundamenta el logro del desarrollo de las competencias científicas en espacios de multiculturalidad, acerca de esto la doctora Yorinay Odreman indica en su investigación doctoral titulada: "Enseñanza de la ciencia ambiental orientada a la valoración de los saberes populares a través de las prácticas artesanales",

...una vez concluida la investigación emergieron significaciones generales desde las propias reflexiones compartidas en un marco filosófico de entorno cultural que propicio discursos individuales y colectivos que se orientaron desde una aproximación dialógica la generación de una propuesta de intervención educativa para los ámbitos formal y no formal atendiendo las temáticas de: Las prácticas artesanales y los saberes populares en el marco de la educación ambiental: valor y uso; diversidad de las prácticas artesanales presentes en la comunidad, revalorizando la identidad cultural local y; utilidad turística de las prácticas artesanales. ( (Odreman Torres, 2016) 
El anterior ejemplo evidencia que desde lo cultural se llega a prácticas educativas democráticas y que revaloran los saberes previos al acto pedagógico como insumos para la formación en competencias científicas pertinentes y requeridas en contextos particulares o especiales. Surge el interrogante entonces de cómo influye el conocimiento y la práctica profesional o el quehacer del maestro para obtener estas experiencias significativas que induzcan el discente a lograr simbiosis entre ciencia y cultura.

Atendiendo a lo antes expuesto se puede indicar que el tipo de pensamiento del maestro o la forma como desarrolla el acto educativo es fundamental para un asertivo proceso de enseñanza aprendizaje, acerca de esto Porlan plantea que la forma "de pensar de los profesores es sumamente importante para la orientación de los procesos y habilidades en el aula de clases". (Porlán, R., Rivero, A y Martín del Pozo, R., 1997)

Existe la convicción en este documento que un maestro no puede dar lo que no tiene, por lo tanto, él es quien en primera instancia anima y orienta la educación científica, particularmente afianza o desarrolla competencias científicas en sus estudiantes, de acuerdo con su formación y concepciones; además ejerce el liderazgo para la transformación educativa a través de sus prácticas en el aula. Por esto es importante observar la necesidad de identificar las concepciones de los docentes acerca del proceso enseñanza-aprendizaje de las ciencias, su pensamiento y conocimiento sobre las competencias científicas en la multiculturalidad. Atendiendo a esto, al PEI (proyecto educativo institucional) y al modelo pedagógico institucional, entre otros, se precisa hacer o no reinducciones a los maestros para que logren apropiarse de los insumos pedagógicos institucionalizados y así se obtenga un trabajo en equipo en términos de las dos referencias conceptúales propósitos de este texto.

En este orden de ideas, lo antes planteado implica que el docente desarrolle un adecuado pensamiento crítico y además se caracterice por una forma de educar en la inclusión y la justicia, solo así podrá abordar escenarios educativos multiculturales propicios para la enseñanza en competencias. La coherencia entre teoría y práctica como también el alejarse de una epistemología reduccionista, mecanicista y memorista y acercarse más bien a una constructivista; son imperativos para alcanzar la idoneidad de un maestro que se considere apto para educar en el nuevo siglo y milenio.

En la actualidad para educar en ciencias es necesario procesos de formación integral que consideren escenarios multiculturales los cuales permitan visibilizar las competencias específicas relacionadas con las ciencias naturales desde diferentes ámbitos muy acordes con el tipo de 
ciudadano universal observado en el siglo XXI.

La formación integral implica un currículo holístico, flexible (Durk, C y Loren, C, 2014) inclusivo, contextualizado y diversificado (Bugueño, 2017) entre otros; existen actualmente varias tendencias curriculares pertinentes para estos tiempos sin embargo dos de ellas sobresalen:

por un lado, una perspectiva amplia que ve el currículo como el producto de un proceso de selección y organización de contenidos relevantes por las características, las necesidades y aspiraciones de la sociedad, y que abarca las finalidades y los objetivos de la educación, los planes y los programas de estudio, la organización de las actividades de enseñanza y de aprendizaje, así como las orientaciones respecto a la evaluación de lo que se ha aprendido. Por otro lado, un enfoque restringido que considera el currículo como el conjunto de programas de estudios construido sobre bases disciplinares, (Gauthier, 2011,2014); (Jonnaert, 2007); (Jonnaert, P., Ettayebi,M., et Opertti, R., 2008)

Circunscribir un currículo observando solo lo disciplinar es muy reductivo, por lo tanto, se considera que éste, debe responder a las preguntas sobre el para qué, el qué, el dónde y el cómo educar; además con pertinencia al contexto y responda a las exigencias o necesidades del grupo social o comunidad educativa donde se encuentre inmersa la institución. El considerar los recursos naturales de la localidad o región, la historia del desarrollo económico de la población son algunos factores decisivos para direccionar un currículo pero también es relevante detenerse en los avances científicos, la movilidad nacional e internacional y fenómenos como la globalización o la internacionalización de la educación, los cuales son referentes importantes para encaminar un tipo de educación particular la cual debe ser caracterizada en un currículo macro regional y en uno micro institucional o local.

Un ejemplo de lo antes expresado es lo que viene haciendo Japón, país que realizó cambios curriculares significativos, disminuyendo el numero de disciplinas e incorporando otras necesarias para la existencia de cualquier ser humano como la economía doméstica también enfatizó sobre tecnología e idiomas (cinco), lo cual permite la formación de un ciudadano cosmopolita y competitivo para este siglo. Acerca de esto el autor (De Zubiría, 2013) en su texto indica: “Cómo diseñar un currículo por competencias" y propone la necesidad de construir currículos no tan segmentados de disciplinas.

El diseñar currículos por competencias, ajustándolos a las disciplinas requeridas para las exigencias del mundo actual y además contemplando la multiculturalidad como elemento emergente de las sociedades del siglo XXI, no es una opción; sencillamente es formar de la 
vida para la vida, como lo estipula un gran eslogan de una institución educativa del municipio de Pueblo viejo, Departamento del Magdalena en Colombia. Se puede indicar también que los currículos fundamentados en una epistemología disciplinar integradora y en el desarrollo de la divergencia como estrategia para el fomento de la creatividad y resolución de problemas; conducen al afianzamiento del pensamiento lógico y complejo por lo cual es pertinente adelantarlo en comunidades educativas como las de los países latinoamericanos caracterizados con altos grados de intolerancia y exclusión o marginación.

Lo axiológico tampoco se puede obviar en un currículo de avanzada identificado por la sinergia entre lo multicultural y lo científico; el respeto como valor universal al igual que la responsabilidad y lo ético serán transversales a cualquiera de las disciplinas que se escojan para formar el plan de estudio de una institución educativa en estos tiempos. Los valores antes mencionados son decisivos para la conformación de equipos de trabajo, y el desarrollo de procesos educativos desde la óptica de una educación por competencias en espacios multiculturales. Competencias científicas como la indagación, identificación y comunicación requieren una alta valoración del respeto en la estructura comportamental de los discentes, los cuales son el centro de interés en el proceso de formación.

Los elementos antes anotados son pertinentes para la construcción de currículos apropiados para la educación del ciudadano del siglo XXI, los cuales deben diseñarse teniendo en cuenta un tópico caracterizador de la cultura local pero también de la regional y la nacional establecido como estrategia para la formación del educando. Obviar documentos guías de tipo nacional como los derechos básicos de aprendizaje (DBA), las mallas curriculares y de aprendizajes, los lineamientos curriculares, los estándares básicos de competencias; sería un error garrafal en la construcción o diseño de un currículo.

Los documentos antes referenciados hay que articularlos con las estrategias didácticas y de multiculturalidad que exige el proceso educativo, en este sentido es importante precisar que cuando se hace referencia a las estrategias didácticas se consideran éstas como "herramientas que facilitan el proceso de enseñanza-aprendizaje" (Orellana, 2017) y al hacer alusión a las competencias multiculturales, se indica que "uno de los objetivos finales más nítidos de la educación multicultural consiste en preparar a todos los alumnos -mayoritarios y, fundamentalmente, minoritarios- para poder comprender, adaptarse y funcionar adecuadamente, tanto en la cultura mayoritaria como en la minoritaria; es decir, generar una auténtica "competencia multicultural”. (James, 1989)Además, formar en competencias multiculturales implica desarrollar en los diversos alumnos conocimientos (sobre las culturas en contacto), habilidades (dominio de las varias lenguas) y actitudes (positivas respecto a la diversidad cultural); cualidades, todas ellas, que les permitan participar, según 
situaciones, necesidades $\mathrm{u}$ opciones, tanto en la cultura mayoritaria como en la minoritaria $\mathrm{u}$ originaria. (Fermoso, 1992)

Atendiendo a los anteriores conceptos se puede inducir que desarrollar competencias didácticas en contextos multiculturales es lograr que el docente tenga la capacidad y habilidad de reconocer los contextos culturales en los cuales están inmersos sus alumnos y a partir de esto seleccione las estrategias adecuadas para que ellos aprehendan los conocimientos atenientes a sus culturas, sean habilidosos en el dominio de lenguas y asuman una posición proactiva respetuosa en la diversidad cultural."

Otro autor relevante para este documento y que hace referencia a las competencias multiculturales es Silvestre:

La competencia multicultural es entendida como aquellos conocimientos, habilidades y actitudes que permiten diagnosticar los aspectos personales y las demandas generadas por la diversidad cultural. Facilitan negociar, comunicarse y trabajar en equipos interculturales $\mathrm{y}$, ha $\neg$ cer frente a las incidencias que surgen en el escenario educativo mediante el autoaprendizaje intercultural y la resolución de problemas que consideren las otras culturas (Terrado, 2016).

Terrado, hace referencia a la multiculturalidad y enfatiza hacia el logro del autoaprendizaje intercultural lo cual implica llegar a lo sublime del multiculturalismo: el interculturalismo; por lo tanto, el maestro competente buscará en gran medida que sus estudiantes interactúen culturalmente y acepten al otro, aunque manifiesten costumbres, valores, entre otros, diferentes.

Atendiendo al marco referencial conceptual expresado hasta ahora, es pertinente observar un país con grandes contrastes en cada uno de los subsistemas de la sociedad: Colombia, para luego encausar el análisis de la temática de este artículo al caribe colombiano.

En la Colombia del siglo XXI, con una gran riqueza cultural donde convergen seis (6) regiones cada una de ellas con unas características propias, pero con una gran población diversa que interactúa simultáneamente en diferentes espacios geográficos; se aprecian dualidades y contrates paradójicos en la convivencia de más de 50.000.000 millones de colombianos. El estado no ha podido solucionar las desigualdades sociales y se incrementa cada día más la pobreza. Acerca de esto el informe de los sabios planteó:

En Colombia las limitaciones del acceso a la educación de amplias capas de la población 
(educación formal, desarrollo de capacidades de los ciudadanos, creación, ciencia, innovación y preparación para el trabajo), y las dificultades para la sostenibilidad con calidad de dichos procesos cuando ellos se logran, afectan el desarrollo humano y están relacionadas, por una parte, con la escasa dinámica de crecimiento de la economía, de la productividad y de la innovación, y por otra, con la precariedad de las dinámicas sociales, políticas y culturales. Estas limitaciones y dificultades tienen sus orígenes en las grandes desigualdades instaladas en nuestro país. A pesar de que la distribución del ingreso tuvo un mejoramiento importante en la última década, Colombia sigue siendo uno de los países más desiguales del mundo. El coeficiente de Gini de 2018 fue de 0.51, situación que afecta diferencialmente de manera negativa los contextos rurales y las poblaciones de más alta vulnerabilidad (sectores rurales, indígenas, mujeres). (Forero, 2019)

Bajo las premisas antes expuestas, se tiene un servicio educativo que de igual forma responde a lo antes descrito con instituciones educativas en situaciones de inequidad en lo concerniente a la diversidad cultural. La educación de las etnias, la población afrocolombiana, entre otros, denominadas minoritarias, han tenido a lo largo de la historia de la educación colombiana menos posibilidades de lograr equiparar indicadores de gestión, eficiencia y eficacia como los de la población mayoritaria.

Por otro lado, una gran parte de los docentes colombianos no están cualificados para desarrollar en el acto educativo, procesos que faciliten la aceptación de la multiculturalidad. En este sentido es muy relativo desarrollar competencias científicas sino se aceptan los saberes tradicionales de cada cultura participante en el aula de clases. Con relación a lo antes expresado lo comisión de sabios-2019, recomienda una restructuración del sistema de formación de maestros y la creación del instituto superior de investigación en educación y alta formación de maestros y redes subregionales de centros de innovación en educación, a través de esta propuesta se busca que los docentes se acerquen mas a las culturas comunitarias y de esta forma logren impactar de mejor forma la educación que brindan a sus educandos; sobre este aspecto la comisión de sabios opina:

Con el propósito de romper el aislamiento de la cultura y de las comunidades que muchos maestros enfrentan, los Centros de Innovación en Educación funcionarán en espacios contiguos a las casas de la cultura en todo el país y efectuarán tareas que vinculen a los maestros con la comunidad, .................es empoderar a los maestros para investigar e innovar de manera sistemática, a la luz de los más avanzados conocimientos científicos, se hace indispensable para mejorar la calidad de la educación y convertirla 
en un instrumento que cierre las brechas de equidad. La posibilidad de transmitir a las nuevas generaciones la curiosidad, el amor por el conocimiento y la búsqueda de soluciones para los problemas del entorno, depende de que los maestros desarrollen su propia sensibilidad y habilidades expresivas, y conozcan el contexto histórico, social y cultural en el que se desenvuelven a través de las artes, las humanidades y las disciplinas científicas. (Forero, 2019, a)

Los gobiernos que ha tenido Colombia en las últimas décadas se han preocupado por legislar en etnoeducación, población afro, entre otros, pero la realidad educativa es otra, persiste la discriminación y la poca aceptación de las culturas minoritarias. Acerca de esto Reales y Berrocal opinan: "Con el actual sistema educativo de Colombia el alumno debe amoldarse a la institución, y lo atinente es lo contrario la institución es la que debe adaptarse a las necesidades de cada uno de sus alumnos" (Reales, R y Berrocal, J, 2019)

No muy ajeno a lo expresado anteriormente, se encuentra el caribe colombiano, el cual en las pruebas que aplica el estado: Pruebas saber $3^{\circ}, 5^{\circ}, 9^{\circ}, 11^{\circ}$ y las ecaes; los resultados sacados por los estudiantes costeños distan y están por debajo de la media nacional.

Es en este contexto caribeño mediante el cual se busca desarrollar competencias científicas a través de escenarios multiculturales. Tanto Colombia y en particular la costa caribe, tienen retos extraordinarios para lograr entronizar un currículo que permita la simbiosis que se ha mencionado en este texto entre desarrollo de competencias científicas y la multiculturalidad. Existe la seguridad de obtener avances en estos caminos, pero se requiere en primeras instancias de unas políticas claras sobre el caso. El maestro requiere cualificación sobre el tema, pero a la vez es fundamental su actitud y aptitud de cambio donde la creatividad para innovar en el acto educativo sea la base para el inicio de nuevas formas de enseñanza donde "articule", la ciencia con cultura; este ejercicio será de vanguardia y ejemplo no solo para el país del café, carbón y la esmeralda sino también para el mundo entero.

\section{CONCLUSIONES}

Los conceptos de competencia y multiculturalidad convergen en un mundo cada día más internacionalizado y sujeto a fenómenos como la globalización, la sociedad del conocimiento y la tecnología. Entre las competencias científicas que más destacan los autores y las organizaciones nacionales e internacionales con validez en las comunidades científicas están: identificar, indagar, explicar, comunicar, trabajar en equipo, entre otras. 
Es fundamental que el docente de ciencias naturales tenga claridad en su desarrollo de pensamiento al igual que los tipos de conocimientos que emplea en el aula para lograr que el discente no entre en procesos de conflictos cognitivos sobre lo que concierne al proceso de aprendizaje de las competencias científicas respectivas.

El currículo en un proceso de desarrollo de competencia bajo experiencia multiculturales también hay que caracterizarlo en procesos flexibles, democráticos, incluyentes y participativos. La práctica profesional indica que en currículos como los antes expuesto surgen tensiones principalmente en lo que respecta al número de disciplinas, organización escolar, formación de docentes, recursos pedagógicos-didácticos, entre otros.

Colombia y en particular la costa caribe, tiene grandes retos en la tarea de lograr una simbiosis entre ciencia y cultura, la comisión de sabios-2019, aporta caminos para esto como la de restructuración del sistema de formación de maestros y la creación del instituto superior de investigación en educación y alta formación de maestros y redes subregionales de centros de innovación en educación.

En síntesis, para formar el hombre del siglo XXI se requiere un docente con pensamiento y conocimientos acorde al siglo de referencia, pero con amplio espectro cognitivo sobre los problemas necesidades de sus comunidades las cuales se encuentran generalmente ligadas a procesos de multiculturalidad, entendido esto como aceptación de la diversidad cultural pluriétnica representada en Colombia por los indígenas, los afrodescendientes, entre otros.

\section{REFERENCIAS BIBLIOGRÁFICAS}

Arteta, J., Chona, G., Fonseca, G., Martínez, S, Ibáñez, S. (2002). Las competencias científicas y el pensamiento de los profesores de Ciencias Naturales. Revista El Oficio de Investigar. Educación y Pedagogía Frente a Nuevos Retos. Colección: Desarrollos en Investigación en Educación. No.3. CIUP. UPN. Bogotá. p.247

Banks, J (1989). Multicultural Education: Traits and Goals, en Multicultural Education. Issues and Perspectives, ed. J.A. Banks y C.A. Banks, (Londres: Allyn and Bacon, 1989), 7.

Baldomero, R. (2016). El saber pedagógico científico del Docente. Una experiencia desde la cotidianidad. Tesis Doctoral, Universidad Santa María, Humanidades y educación, Barquisimeto. 
Bernabé, M. (2012). Bernabé, M. (2012). Pluriculturalidad, multiculturalidad e interculturalidad, conocimientos. Revista Educativa Hekademos,11, Año, junio 2012. Pp.67-76. ISSN:1989-3558

Bugueño, H. (2017). La indagación científica: Una estrategia para aprender colaborativamente ciencias naturales en la Educación Primaria. Tesis Doctoral, Universidad de Alcalá, Educación, España.

Brent Shields, B y Molen, G (productores) y Bleckner. J (director). (2011). Detrás de la Pizarra. Nobody Don't love Nobody (Nadie los ama, nadie). EE. UU. Studio Universal

De Zubiría, J. (2013). ¿Cómo diseñar un currículo por competencias? Fundamentos, lineamientos y estrategias. Bogotá DC: 2da. Edición. Ed. Magisterio. Pp. 1-271. ISBN: 978-201112-3

Duk, C. y Loren, C. (2014). Flexibilización del currículum para atender la diversidad. Revista Latinoamericana de Educación Inclusiva, 4(1), Pp. 187-210. Tomado de: http://www.rinace.net/ rlei/numeros/vol4-num1/art9.pdf

Fagade Lean, J. (2015). Multiculturalismo: avances en la sociedad. Revista de Educación, $14-21$.

Fermoso, P. (1992). Formación del profesorado para la educación multicultural, en Educación intercultural, ed. P. Fermoso (Madrid: Narcea, 1992), p. 22

Forero, C. (2019). Colombia hacia la sociedad del conocimiento. Informe de la misión internacional de sabios 2019 por la educación, la ciencia, la tecnología y la innovación. Versión preliminar No 1

Forero, C. (2019, a). Colombia hacia la sociedad del conocimiento. Informe de la misión internacional de sabios 2019 por la educación, la ciencia, la tecnología y la innovación. Versión preliminar No 1

Gauthier, R.F. (2011). Le curriculum dans les politiques educatives- (Dossier). Revue Internacional d'education de Sèvres 56. 31-165. Tomado de: https://journals.openedition.org/ ries/1013 
Jonnaert, P. 2007. Le concept de competence revisite. Montreal: Universite du Quebec a Montreal.

Jonnaert, P., Ettayebi, M. et Opertti. R. (2008). Dinamarqués des reformes éducatives contemporaines. Pp. 17-25 en Logique de compétences et développement curriculaire, edité par les auteurs. Brussels: De Boeck.

Maldonado, B. (2016). Hacia un país plural: educación comunitaria en Oaxaca frente a la política de interculturalidad cero. LiminaR. Estudios Sociales y Humanísticos, vol. XIV, núm. 1, pp. 47-59, ISSN: 1665-8027. Disponible en: https://www.redalyc.org/articulo.oa?id=745/74543269004

Murgas, S. (2015). El concepto de competencia en el desarrollo de la educación: Origen y evolución. Madrid: E-ducare.

Odreman Torres, Y. (2016). Ciencia ambiental no formal orientada a la valoración de los saberes populares a través de las prácticas artesanales. Tesis individual, Universidad Pedagógica Experimental Libertador, Educación, Barinas.

Orellana, C. (2017). Las estrategias didácticas y su uso dentro del proceso enseñanza y aprendizaje en el contexto de las bibliotecas escolares. Vol. 7 No.1. DOI: 101551/eci.v7il.27241

Porlán, R., Rivero, A y Martín Del Pozo, R. (1997). Conocimiento profesional y epistemología de los profesores I teoría, métodos e instrumentos. Enseñanza de las ciencias: revista de investigación y experiencias didácticas, ISSN 0212-4521, ISSN-e 2174-6486, Vol. 15, Nº 2, 1997, págs. 155-171

Tomado de: https://dialnet.unirioja.es/servlet/articulo? codigo $=94881$

Reales, R y Berrocal, J. (2019). Educación inclusiva en Colombia y la Región del Caribe colombiano: Estrategias para mostrar1. Revista Legem, Universidad del Atlántico 5 (1), Pp. 93-123 ISSN: $2346-2787$

Terrado Silvestre, C. (2016). La multiculturalidad de saberes educativos: Un enfoque transdisciplinario. Tesis, Universidad Central de Venezuela, Caracas. 